TITLE:

\title{
Hybrid density functional study of oxygen vacancies in $\mathrm{KTaO} 3$ and $\mathrm{NaTaO} 3$
}

AUTHOR(S):

Choi, Minseok; Oba, Fumiyasu; Tanaka, Isao

CITATION:

Choi, Minseok ... [et al]. Hybrid density functional study of oxygen vacancies in KTaO3 and NaTaO3. PHYSICAL REVIEW B 2011, 83(21): 214107.

\section{ISSUE DATE:}

2011-06

URL:

http://hdl.handle.net/2433/161786

RIGHT:

(C2011 American Physical Society 
PHYSICAL REVIEW B 83, 214107 (2011)

\title{
Hybrid density functional study of oxygen vacancies in $\mathrm{KTaO}_{3}$ and $\mathrm{NaTaO}_{3}$
}

\author{
Minseok Choi* and Fumiyasu $\mathrm{Oba}^{\dagger}$ \\ Department of Materials Science and Engineering, Kyoto University, Sakyo, Kyoto 606-8501, Japan
}

Isao Tanaka

Department of Materials Science and Engineering, Kyoto University, Sakyo, Kyoto 606-8501, Japan, Nanostructures Research Laboratory, Japan Fine Ceramics Center, Atsuta, Nagoya 456-8587, Japan, and Fukui Institute for Fundamental Chemistry, Kyoto University, Sakyo, Kyoto 606-8103, Japan

(Received 7 March 2011; published 10 June 2011)

\begin{abstract}
Using the Heyd-Scuseria-Ernzerhof hybrid functional, we systematically study the energetics and electronic properties of oxygen vacancies in $\mathrm{KTaO}_{3}$ and $\mathrm{NaTaO}_{3}$. The oxygen vacancies in these systems show similar behavior. The $2+$ charge state is the most stable for most positions of the Fermi level inside the band gap. The neutral and + charge states become comparable in formation energy with the $2+$ charge state when the Fermi level is close to the conduction band minimum. Therefore, the oxygen vacancies are double shallow donors, which can provide carrier electrons. Two types of off-symmetric configurations, in which the two nearest tantalum atoms of the oxygen vacancies are asymmetrically located, also possibly form as metastable configurations. These metastable configurations show a striking difference in electronic structure from each other; one configuration has a delocalized characteristic as in the case of the stable configuration, while the other induces a deep, localized state in the band gap. On the basis of the predicted formation energies and electronic properties, the previous experimental and theoretical findings relevant to the oxygen vacancies are discussed.
\end{abstract}

DOI: $10.1103 /$ PhysRevB.83.214107

PACS number(s): 61.72.J-, 61.72.Bb, 71.55.Ht

\section{INTRODUCTION}

$\mathrm{KTaO}_{3}$ is classified as an incipient ferroelectric and its high permittivity and low dielectric loss are promising for applications in microwave device. ${ }^{1-3}$ It exhibits a large Seeback coefficient when electrons are introduced by dopants, which is of importance to thermoelectric devices. ${ }^{4,5} \mathrm{NaTaO}_{3}$ is one of the candidates for high-efficient photocatalyst activating for water splitting into $\mathrm{H}_{2}$ and $\mathrm{O}_{2}$ under ultraviolet light irradiation. ${ }^{6}$ In particular, La-doped $\mathrm{NaTaO}_{3}$ has shown good stability and high photocatalytic activity. ${ }^{6}$

The oxygen vacancy $\left(V_{\mathrm{O}}\right)$ is expected to give significant influences to such optical and electrical properties of $\mathrm{KTaO}_{3}$ and $\mathrm{NaTaO}_{3}$, since $V_{\mathrm{O}}$ is known to form with a substantial concentration in these oxides. Therefore, correct understanding of the energetics and structural and electronic properties of $V_{\mathrm{O}}$ is of importance, and the state-of-the-art first-principles calculations can be useful. To date, however, only a few first-principles studies have been reported on $V_{\mathrm{O}}$ in $\mathrm{KTaO}_{3}$ (Ref. 7) and $\mathrm{NaTaO}_{3}$ (Ref. 8) using the generalized gradient approximation (GGA), showing severe band-gap underestimation. Information on the formation energy of $V_{\mathrm{O}}$ and its electronic levels is very limited, in particular for $\mathrm{KTaO}_{3}$.

Likewise, $V_{\mathrm{O}}$ in these oxides has been investigated by experiments, but there exists controversy about its stable charge state. $V_{\mathrm{O}}^{+}$was suggested to be stable in $n$-type systems in a wide range of temperatures from electric conductivity measurements, ${ }^{9}$ whereas electron paramagnetic resonance (EPR) experiments have not clearly proven the existence of $V_{\mathrm{O}}^{+} \cdot{ }^{10-12}$

In this paper, we present a systematic study of $V_{\mathrm{O}}$ in $\mathrm{KTaO}_{3}$ and $\mathrm{NaTaO}_{3}$ using the Heyd-Scuseria-Ernzerhof (HSE06) hybrid functional. ${ }^{13-15}$ An accurate description of the energetics and electronic structure of $V_{\mathrm{O}}$ is our goal, and the HSE06 hybrid functional approach is chosen because it has shown a significant improvement for describing point defects or impurities in wide band-gap semiconductors over the standard density functional approaches. ${ }^{16-20}$ Using the HSE06 functional, the lattice constants and electronic band structure of the perfect $\mathrm{KTaO}_{3}$ and $\mathrm{NaTaO}_{3}$ crystals are examined. Then the atomic configurations and energetics of $V_{\mathrm{O}}$ in these systems are investigated. Finally, the previous experimental and theoretical findings on $V_{\mathrm{O}}$ are discussed on the basis of our results.

\section{COMPUTATIONAL METHODS}

The calculations were conducted using the projector augmented-wave method ${ }^{21}$ as implemented in the VASP code. $^{22-24}$ The standard exchange mixing containing $25 \%$ of Hartree-Fock and 75\% of the Perdew-Burke-Ernzerhof (PBE)GGA (Ref. 25) and the screening parameter of $0.208 \AA^{-1}$ (Ref. 15) were employed in the HSE06 functional. The projector augmented-wave data sets with radial cutoffs of $1.2 \AA(\mathrm{K}), 1.2 \AA(\mathrm{Na}), 1.5 \AA(\mathrm{Ta})$, and $0.8 \AA(\mathrm{O})$ were used. The electronic wave functions were described using a plane wave basis set with an energy cutoff of $400 \mathrm{eV}$. A 135-atom supercell and the $\Gamma$-only $k$-point sampling were used. The atomic coordinates were relaxed until the Hellmann-Feynman force acting on each atom was reduced to less than $0.05 \mathrm{eV} \AA^{-1}$. In all calculations for $V_{\mathrm{O}}$, the effects of spin polarization were included.

The formation energy of $V_{\mathrm{O}}$ is determined by

$$
\Delta E_{f}\left(V_{\mathrm{O}}^{q}\right)=E_{\mathrm{T}}\left(V_{\mathrm{O}}^{q}\right)-E_{\mathrm{T}}(\mathrm{H})+\mu_{\mathrm{O}}+q\left(E_{\mathrm{VBM}}+\varepsilon_{\mathrm{F}}\right),
$$

where $E_{\mathrm{T}}\left(V_{\mathrm{O}}^{q}\right)$ is the total energy of a $\mathrm{KTaO}_{3}$ or $\mathrm{NaTaO}_{3}$ supercell with one $V_{\mathrm{O}}$ in charge state $q(0,+$, and $2+)$, and $E_{\mathrm{T}}(\mathrm{H})$ is the total energy of the host supercell. $\mu_{\mathrm{O}}$ is the oxygen chemical potential, varying between the oxygen-rich 
limit $\left(\mu_{\mathrm{O}}=\frac{1}{2} \mu_{\mathrm{O}_{2} \text { (molecule) }}\right)$ and the oxygen-poor limit $\left(\mu_{\mathrm{Ta}}=\right.$ $\mu_{\mathrm{Ta}(\text { bulk) }}$ and $\mu_{\mathrm{Ta}}+2 \mu_{\mathrm{O}}=\mu_{\mathrm{TaO}_{2} \text { (bulk) }}$ ). These equilibrium conditions are taken on the basis of previous considerations. ${ }^{7,8}$ $E_{\mathrm{VBM}}$ and $\varepsilon_{\mathrm{F}}$ are the valence band maximum (VBM) and the Fermi level measured from the VBM, respectively.

In order to complement the calculations using the HSE06 hybrid functional and finite-sized periodic supercells, some postcorrection processes were carried out. These were carefully applied to defect formation energies with consideration of the characteristics of single-particle states induced by $V_{\mathrm{O}}: 8,26-28$

(i) VBM alignment was applied to charged systems using average electrostatic potentials. In the momentum-space approach with periodic supercells, the total energies of charged systems are evaluated by implicitly introducing uniform compensation-charge to keep the charge neutrality. ${ }^{29}$ This leads to an ill-defined shift of single-particle energy levels. Therefore, it is necessary to line up $E_{\mathrm{VBM}}$ between the host and defective supercells containing non-neutral $V_{\mathrm{O}}$. For this purpose, average electrostatic potentials in a host (defect-free) supercell and those in a bulklike environment far from $V_{\mathrm{O}}$ in defective supercells were used.

(ii) The HSE06 hybrid functional with the standard exchange mixing provides, in general, much better description of the electronic structure of semiconductors and insulators than the GGA or the local density approximation (LDA). However, the HSE06 band gaps $\left(E_{\mathrm{g}}^{\mathrm{HSE06}}\right)$ of $\mathrm{KTaO}_{3}$ and $\mathrm{NaTaO}_{3}$ are still smaller compared with the experimental band gaps $\left(E_{\mathrm{g}}^{\exp }\right)$, as shown in Table I. Therefore, band-gap corrections must be considered to evaluate the formation energies accurately. The corrections were performed on the basis of the characteristics of $V_{\mathrm{O}}$-induced electronic states. Since we found that $V_{\mathrm{O}}$ creates its single-particle states resonant inside the conduction band, the formation energies of $V_{\mathrm{O}}$ were extrapolated to $E_{\mathrm{g}}^{\exp }$ by adding $m \Delta E_{\mathrm{g}}$, where $m$ and $\Delta E_{\mathrm{g}}$ are the number of electrons in the conduction bandlike state released from $V_{\mathrm{O}}\left(m=2\right.$ for $V_{\mathrm{O}}^{0}$ and $m=1$ for $\left.V_{\mathrm{O}}^{+}\right)$and the band-gap difference, equal to $E_{\mathrm{g}}^{\mathrm{exp}}-E_{\mathrm{g}}^{\mathrm{HSE} 06}$, respectively.

(iii) The formation energy of a defect is dependent upon supercell size owing to spurious interactions between defect and its periodic images. For charged defects in ionic crystals, electrostatic interactions are dominant in many cases, as pointed out by Leslie and Gillan ${ }^{30}$ and by Makov and Payne. ${ }^{31}$ It has been reported that such a cell-size dependence can be

TABLE I. Lattice constant, $a(\AA)$, and electronic band gap, $E_{\mathrm{g}}$ (eV). HSE06, GGA, and exp in superscript denote the HSE06, PBEGGA, and experimental values, respectively. Experimental data are collected from Refs. 7 and 8 and references therein.

\begin{tabular}{lcc}
\hline \hline & $\mathrm{KTaO}_{3}$ & $\mathrm{NaTaO}_{3}$ \\
\hline$a^{\mathrm{HSE} 06}$ & 3.995 & 3.947 \\
$a^{\mathrm{GGA}}$ & 4.027 & 3.976 \\
$a^{\exp }$ & 3.988 & 3.929 \\
$E_{\mathrm{g}}^{\mathrm{HSE} 06}$ & 3.40 & 3.60 \\
$E_{\mathrm{g}}^{\mathrm{GGA}}$ & 2.06 & 2.22 \\
$E_{\mathrm{g}}^{\exp }$ & 3.7 & 4.1 \\
\hline \hline
\end{tabular}

affected by characteristics of defect-induced electronic states and also by elastic interactions. ${ }^{16,32-35}$ In this case, a simple use of the Makov-Payne correction scheme ${ }^{31}$ cannot be justified. One practical way to reduce uncertainty arising from the cell-size dependence is to use the correction terms obtained by fitting formation energies calculated using supercells with various sizes to an appropriate formula. However, the HSE06 calculations need too high computational costs to follow this scheme. Instead, one can predict the cell-size dependence on the basis of the GGA or LDA results. For $\mathrm{NaTaO}_{3}$, our previous GGA study found that the formation energies of $V_{\mathrm{O}}^{0}, V_{\mathrm{O}}^{+}$, and $V_{\mathrm{O}}^{2+}$ decrease as the supercell size increases, and such a trend is almost identical for all the charge states. ${ }^{8}$ As a consequence, thermodynamical transition levels obtained using a 135-atom cell are unchanged for a larger supercell containing 625 atoms, and $V_{\mathrm{O}}^{2+}$ is the most stable for most Fermi level position in the band gap. Similar tendencies have been reported on $V_{\mathrm{O}}$ in other semiconducting oxides such as $\mathrm{SrTiO}_{3}$ (Ref. 36) and $\mathrm{TiO}_{2} .{ }^{18}$ The formation energies of $V_{\mathrm{O}}$ in $\mathrm{KTaO}_{3}$ and $\mathrm{NaTaO}_{3}$ by the HSE06 calculations are likely to have the same trend as those by the GGA, ${ }^{8}$ since the HSE06 calculations show single-particle electronic structures similar to the GGA results for $V_{\mathrm{O}}^{0}, V_{\mathrm{O}}^{+}$, and $V_{\mathrm{O}}^{2+}$, as described in Sec. III. Therefore, our discussion and conclusions are unlikely to be altered by the supercell size.

\section{RESULTS AND DISCUSSION}

Figure 1 shows the electronic band structures of pure $\mathrm{KTaO}_{3}$ and $\mathrm{NaTaO}_{3}$ obtained using the HSE06 and PBE-GGA functionals. The computed lattice constants and band gaps are summarized in Table I. As expected, the HSE06 band gaps are larger and much closer to the experimental data than the PBE-GGA values. Band dispersions near the band gap in $\mathrm{KTaO}_{3}$ and $\mathrm{NaTaO}_{3}$ resemble each other. Also in each system, the band dispersions of the HSE06 and PBE-GGA results are quite similar. In all the cases, the conduction band minimum (CBM) and VBM are located at the $\Gamma$ - and $R$-points, respectively. The HSE06 hybrid functional also reproduces the lattice constants very accurately, while the PBE-GGA overestimates by nearly $1 \%$.

Moving to $V_{\mathrm{O}}$ in $\mathrm{KTaO}_{3}$ and $\mathrm{NaTaO}_{3}$, the atomic configurations of $V_{\mathrm{O}}$ are systematically explored. Our calculations indicate that the formation of $V_{\mathrm{O}}$ leads to the nearest Ta atoms relaxed equally and outwardly from their ideal positions in the perfect system. As a result, $V_{\mathrm{O}}$ possesses the atomic configuration with $D_{4 h}$ point-group symmetry, whose principal rotation axis is along the $\mathrm{Ta}-V_{\mathrm{O}}-\mathrm{Ta}$ chain, as depicted in Figs. 2(a) and 2(b). Table II shows the calculated interatomic distance between $V_{\mathrm{O}}$ and its nearest two Ta atoms $\left(d_{V_{\mathrm{O}}-\mathrm{Ta}}\right)$. The three charge states exhibit nearly the same atomic configurations, which is attributed to the delocalized characteristic of $V_{\mathrm{O}}$ in single-particle electronic structure as discussed later.

The formation energies of $V_{\mathrm{O}}$ in $\mathrm{KTaO}_{3}$ and $\mathrm{NaTaO}_{3}$ as a function of the Fermi level are illustrated in Fig. 3. The $2+$ charged $V_{\mathrm{O}}$ is the most stable in most of the Fermi level positions inside the band gap. This indicates that $V_{\mathrm{O}}$ is a double shallow donor in both systems and releases two electrons. At the oxygen-rich limit, the formation energies of $V_{\mathrm{O}}$ are as high as $\sim 6 \mathrm{eV}$. However, under the oxygen-poor limit, the 




FIG. 1. (Color online) Band structure of $\mathrm{KTaO}_{3}$ (top) and $\mathrm{NaTaO}_{3}$ (bottom). Solid (red) and dash-dotted (black) curves denote the HSE06 and PBE-GGA results, respectively. The VBM is set to zero in each case.

formation energies become lower by $3.97 \mathrm{eV}$, corresponding to half the formation energy of $\mathrm{TaO}_{2}$. This amount of change is close to that of the previous GGA results. ${ }^{7,8}$ It leads to the formation energies of $V_{\mathrm{O}}^{2+}$ for the Fermi level at the CBM, $\Delta E_{f}^{\mathrm{CBM}}\left(V_{\mathrm{O}}^{2+}\right)=2.08$ and $1.63 \mathrm{eV}$ for $\mathrm{KTaO}_{3}$ and $\mathrm{NaTaO}_{3}$,
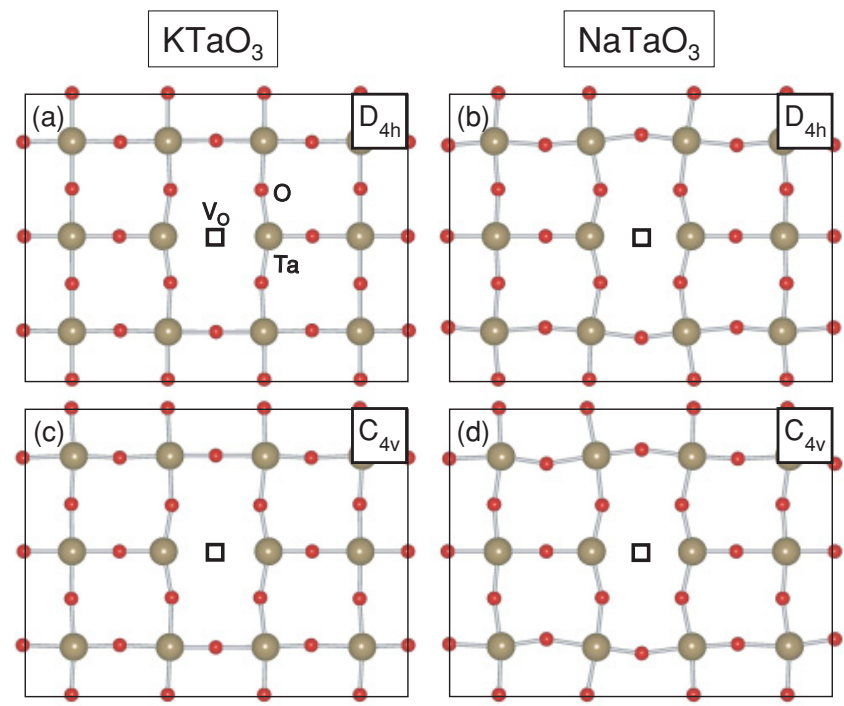

FIG. 2. (Color online) Atomic configurations of neutral $V_{\mathrm{O}}$ in $\mathrm{KTaO}_{3}$ and $\mathrm{NaTaO}_{3}$. Top and bottom panels show the stable $\left(D_{4 h}\right)$ and metastable $V_{\mathrm{O}}-\mathrm{I}\left(C_{4 v}\right)$ configurations, which are illustrated on the Ta-O plane including $V_{\mathrm{O}}$. The principal rotation axis of the $D_{4 h}$ and $C_{4 v}$ point-group symmetry is along the Ta- $V_{\mathrm{O}}-\mathrm{Ta}$ chain.
TABLE II. Atomic distance between $V_{\mathrm{O}}$ and the nearest Ta atoms in $\AA$ units. $D_{4 h}, \mathrm{I}\left(C_{4 v}\right)$, and II $\left(C_{4 v}\right)$ denote the stable $V_{\mathrm{O}}$, metastable $V_{\mathrm{O}}$-I, and metastable $V_{\mathrm{O}}$-II configurations, respectively. Ideal denotes the interatomic distance between $\mathrm{O}$ atom and its nearest $\mathrm{Ta}$ atoms in the perfect-crystal cell.

\begin{tabular}{lrrrrrrr}
\hline \hline & \multicolumn{3}{c}{$\mathrm{KTaO}_{3}$} & & \multicolumn{3}{c}{$\mathrm{NaTaO}_{3}$} \\
\cline { 2 - 3 } \cline { 6 - 8 } & $D_{4 h}$ & $\mathrm{I}\left(C_{4 v}\right)$ & $\mathrm{II}\left(C_{4 v}\right)$ & & $D_{4 h}$ & $\mathrm{I}\left(C_{4 v}\right)$ & $\mathrm{II}\left(C_{4 v}\right)$ \\
\hline Ideal & & 2.00 & & & 1.97 & \\
$V_{\mathrm{O}}^{0}$ & 2.18 & $1.80,2.56$ & $1.70,2.48$ & & 2.08 & $1.78,2.36$ & $1.61,2.30$ \\
$V_{\mathrm{O}}^{+}$ & 2.19 & $1.80,2.56$ & $1.70,2.49$ & & 2.08 & $1.78,2.36$ & $1.60,2.31$ \\
$V_{\mathrm{O}}^{2+}$ & 2.19 & $1.80,2.56$ & $1.80,2.56$ & & 2.08 & $1.78,2.37$ & $1.78,2.37$ \\
\hline
\end{tabular}

respectively. Hence, $V_{\mathrm{O}}$ is likely to form under oxygen-poor conditions and may contribute to the physical properties such as the $n$-type conductivity in $\mathrm{KTaO}_{3}$ and $\mathrm{NaTaO}_{3}$. In the singleparticle electronic structure, hydrogenic effective-mass states are found in the neutral charge state, indicating that $V_{O}^{0}$ is regarded as $V_{\mathrm{O}}^{2+}$ with weakly bound or unbound two electrons. Similarly, $V_{\mathrm{O}}^{+}$corresponds to $V_{\mathrm{O}}^{2+}$ that has a weakly bound or unbound electron. These features are compatible with the formation-energy diagram shown in Fig. 3, indicating nearly the same formation energies for $V_{\mathrm{O}}^{0}, V_{\mathrm{O}}^{+}$, and $V_{\mathrm{O}}^{2+}$ when the Fermi level lies near the CBM.
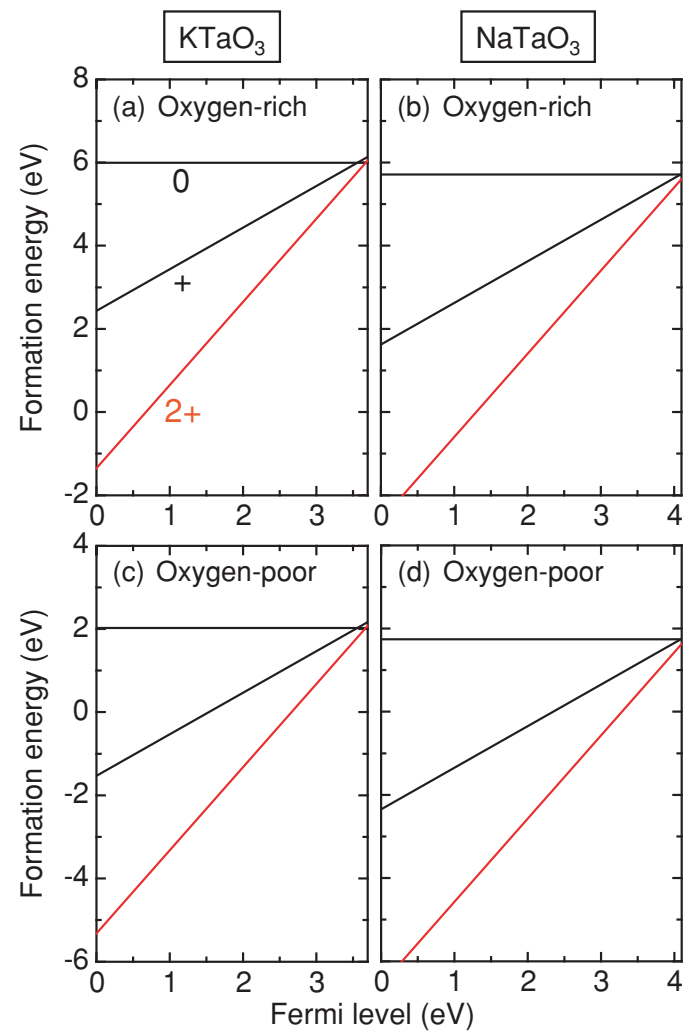

FIG. 3. (Color online) Formation energies of $V_{\mathrm{O}}$ in $\mathrm{KTaO}_{3}$ (left) and $\mathrm{NaTaO}_{3}$ (right) as a function of the Fermi level. Top and bottom panels indicate the formation energies at the oxygen-rich and oxygenpoor limits, respectively. The slope corresponds to the charge state as defined by Eq. (1). Note that in these systems the $2+$ charge state is stable for most positions of the Fermi level in the band gap. 

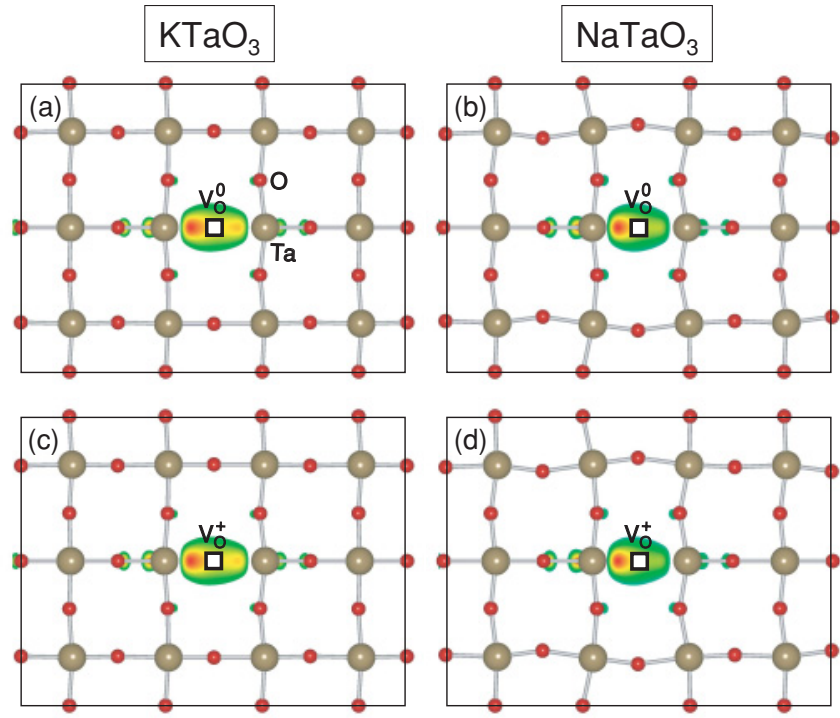

FIG. 4. (Color online) Squared wave functions $\left(\left|\psi_{\mathrm{e}}\right|^{2}\right)$ at the occupied in-gap single-particle states in the majority spin component of metastable $V_{\mathrm{O}}$-II $\left(C_{4 v}\right)$ configurations in $\mathrm{KTaO}_{3}$ and $\mathrm{NaTaO}_{3}$. Any localized in-gap states are not found in the minority spin component. Panels (a) and (b) correspond to $\left|\psi_{\mathrm{e}}\right|^{2}$ for the deeper state of $\mathrm{V}_{\mathrm{O}}^{0}$. Panels (c) and (d) show $\left|\psi_{\mathrm{e}}\right|^{2}$ of $V_{\mathrm{O}}^{+}$. More details are described in the text. $\left|\psi_{\mathrm{e}}\right|^{2}$ are illustrated on the Ta-O plane including $V_{\mathrm{O}}$, which are the cross-sections of isosurfaces at $>0.03 \AA^{-3}$ (Ref. 37).

The $D_{4 h}$ point-group symmetry has been typically considered for the atomic configuration of $V_{\mathrm{O}}$ in various cubic perovskite oxides. Here we examine the effects of local atomic distortion near $V_{\mathrm{O}}$ to break symmetry, and find that a configuration with a lower symmetry, $C_{4 v}$, is also energetically preferable for $V_{\mathrm{O}}\left(V_{\mathrm{O}}-\mathrm{I}\right)$. As shown in Figs. 2(c) and 2(d), $V_{\mathrm{O}}$-I has asymmetric $d_{V_{\mathrm{O}}-\mathrm{Ta}}$, differently from that in the stable $D_{4 h}$ symmetric configuration. The computed $d_{V_{\mathrm{O}}-\mathrm{Ta}}$ values are summarized in Table II. In the single-particle electronic structure, $V_{\mathrm{O}}$-I shows a spatially delocalized electron wave function, which resembles that of the stable $D_{4 h}$ configuration. Therefore, $V_{\mathrm{O}}-\mathrm{I}$ is also a double shallow donor.

Another configuration of $V_{\mathrm{O}}\left(V_{\mathrm{O}}-\mathrm{II}\right)$ is found, which forms electron-occupied single-particle states inside the band gap. For the neutral charge state, there exist the states at 0.26 and $0.83 \mathrm{eV}$ for $\mathrm{KTaO}_{3}$ and 0.36 and $0.89 \mathrm{eV}$ for $\mathrm{NaTaO}_{3}$ below the CBM. The shallower state in each system is characterized by a host CBM-like orbital with a spatially delocalized squared wave function $\left(\left|\psi_{\mathrm{e}}\right|^{2}\right)$. Differently, the deeper state exhibits a strongly localized $\left|\psi_{\mathrm{e}}\right|^{2}$ around $V_{\mathrm{O}}$ as illustrated in Figs. 4(a) and $4(\mathrm{~b})$. In the + charge case, localized states are found at 0.69 and $0.76 \mathrm{eV}$ below the $\mathrm{CBM}$ in $\mathrm{KTaO}_{3}$ and $\mathrm{NaTaO}_{3}$, respectively. As seen in Figs. 4(c) and 4(d), these states have orbital characteristics similar to the deeper states of the neutral case, that is, those at $0.83 \mathrm{eV}\left(\mathrm{KTaO}_{3}\right)$ and $0.89 \mathrm{eV}\left(\mathrm{NaTaO}_{3}\right)$ below the CBM.

From the viewpoint of the formation energy, $\Delta E_{f}\left(V_{\mathrm{O}}^{0}\right)$ of $V_{\mathrm{O}}$-I and $V_{\mathrm{O}}$-II are higher than that of the stable $V_{\mathrm{O}}$, that is, the $D_{4 h}$ configuration, by 0.27 and $0.35 \mathrm{eV}$ for $\mathrm{KTaO}_{3}$ and 0.44 and $0.69 \mathrm{eV}$ for $\mathrm{NaTaO}_{3}$, respectively. Therefore, $V_{\mathrm{O}}$-I and $V_{\mathrm{O}}$-II are metastable and may form only in specimens grown by conditions that are far from thermal equilibrium and/or after specific post-treatments. Note that the total energies of $V_{\mathrm{O}}$-I and $V_{\mathrm{O}}$-II are, in fact, lower than that of the stable $V_{\mathrm{O}}$. However, we found that the asymmetric relaxation to break the cubic symmetry, as occurring for $V_{\mathrm{O}}$-I and $V_{\mathrm{O}}$-II in $C_{4 v}$ symmetry, also lowers the total energy of the perfect crystal cell for both $\mathrm{KTaO}_{3}$ and $\mathrm{NaTaO}_{3}$. The distorted perfect crystal is therefore used for the evaluation of the formation energies of $V_{\mathrm{O}}-\mathrm{I}$ and $V_{\mathrm{O}}$-II to compensate the energy gain associated with the asymmetric relaxation. It is also noteworthy that $V_{\mathrm{O}}-\mathrm{I}$, whose $\left|\psi_{\mathrm{e}}\right|^{2}$ is similar to that of the stable $V_{\mathrm{O}}$, has a lower energy than $V_{\mathrm{O}}$-II showing localized $\left|\psi_{\mathrm{e}}\right|^{2}$ characteristics. This supports our conclusion that $V_{\mathrm{O}}$ acts as a shallow double donor.

Next, we discuss the physical properties relevant to $V_{\mathrm{O}}$ for each system on the basis of our results:

(i) $\mathrm{KTaO}_{3}$. Through the temperature-dependence measurements of electric conductivity, the ionization energy of $V_{\mathrm{O}}^{+}$ in $\mathrm{KTaO}_{3}$ was suggested to be $0.1-0.2 \mathrm{eV} .{ }^{9}$ Laguta and coworkers reported through thermally stimulated conductivity measurements that the concentrations of shallow-electron traps, assigned as $V_{\mathrm{O}}^{0}$ or $V_{\mathrm{O}}^{+}$states, are $10^{11}-10^{13} \mathrm{~cm}^{-3}$, but the EPR could not detect $V_{\mathrm{O}}$ in the same samples. ${ }^{11}$ They attributed no such EPR signal to (i) a lower concentration of $V_{\mathrm{O}}$ than the EPR sensitivity $\left(10^{15}-10^{16} \mathrm{~cm}^{-3}\right)$, (ii) a large radius of $V_{\mathrm{O}}$-induced states at the traps, and/or (iii) the coupling of many of $V_{\mathrm{O}}$ with a metal impurity, leading to deeper electron traps. As predicted by our calculations, the double shallow donor nature of $V_{\mathrm{O}}$ can be an actual cause of no EPR signal.

The local polar microregions have been observed in nominally pure $\mathrm{KTaO}_{3}$ samples, ${ }^{38,39}$ and $\mathrm{Ta}^{4+}-V_{\mathrm{O}}^{2+}$ or $\mathrm{Ta}^{4+}$ $V_{\mathrm{O}}^{2+}-\mathrm{Me}_{I}^{4+}$ centers were proposed to explain them, where $\mathrm{Me}_{I}$ is an unintentionally incorporated metal impurity in the given samples. ${ }^{12,40,41}$ Within these paramagnetic centers, a Ta atom and a metal impurity capture electrons released from the nearest $V_{\mathrm{O}}$, leading to the inversion symmetry breaking and thereby to the local dipole moment. However, $V_{\mathrm{O}}^{+}$is unlikely to form under thermodynamical equilibrium, according to our results and also to the results of previous atomistic simulations using empirical interatomic potentials and a shell model. ${ }^{42}$ In addition, the stable $V_{\mathrm{O}}$ configurations have $D_{4 h}$ symmetry, which cannot be a source of polarization. Therefore, thermodynamically stable $V_{\mathrm{O}}$ is not responsible for the observations. However, the metastable $V_{\mathrm{O}}$-II configuration breaks the inversion symmetry, and thereby may produce the polarization when this configuration can form with a high-enough concentration under some specific conditions.

Similar observations in $\mathrm{SrTiO}_{3}$ may give a hint to resolve this issue. A ferroelectric state with oxygen deficiency, ${ }^{43}$ and local polar nanoregions and relaxorlike ferroelectricity have recently been found in bulk and thin films of $\mathrm{SrTiO}_{3}$ with composition of $\mathrm{Sr} / \mathrm{Ti}<1 .{ }^{44}$ A recent theoretical study proposed that $\mathrm{Ti}$ antisite defects, which are composed of a $\mathrm{Ti}$ atom off-centered from the $\mathrm{Sr}$ site, can be promising candidates for the non-stoichiometry-induced ferroelectricity in $\mathrm{SrTiO}_{3}{ }^{45}$ These defects have local electric dipoles and are energetically favorable to form under Ti-rich conditions. By analogy, we propose that Ta antisite defects, that is, a Ta atom off-centered from the $\mathrm{K}$ site, are also considerable sources of the polar microregions. In fact, it has been reported that extrinsic A-site dopants such as Li can create the local polar clusters through 
a large off-centering $(\sim 1 \AA)$ from the $\mathrm{K}$ site and thereby produce the relaxorlike ferroelectric state. ${ }^{46,47}$ In the same sense, Ta antisite defects are expected to play roles as intrinsic off-centered A-site dopants.

(ii) $\mathrm{NaTaO}_{3}$. To our knowledge, there is only one computational study reported dealing with the structural and electronic properties of $V_{\mathrm{O}}$ as well as other native defects in $\mathrm{NaTaO}_{3} \cdot{ }^{8}$ This study was conducted using the PBE-GGA functional, and showed that $V_{\mathrm{O}}^{2+}$ is thermodynamically the most stable for most positions of the Fermi level inside the band gap. This finding on $V_{\mathrm{O}}$ is the same as what the present HSE06 calculations figure out. In other words, both studies suggest that $V_{\mathrm{O}}$ acts as a double shallow donor in $\mathrm{NaTaO}_{3}$, providing carrier electrons. Even at the oxygen-rich limit, where $V_{\mathrm{O}}$ is energetically unfavorable in general, the HSE06 formation energy of $V_{\mathrm{O}}$ is largely negative when the Fermi level is located near the VBM [see Fig. 3(b)]. Such a negative $\Delta E_{f}$ implies that making $p$-type $\mathrm{NaTaO}_{3}$ is difficult under thermal equilibrium because of the spontaneous formation of $V_{\mathrm{O}}^{2+}$ and hence carrier compensation. No EPR measurement has been reported about $V_{\mathrm{O}}$ in $\mathrm{NaTaO}_{3}$.

In connection with the theoretical approach, one thing is noteworthy. As mentioned above, our HSE06 calculations in conjunction with the band-gap correction yielded a $\Delta E_{f}^{\mathrm{CBM}}\left(V_{\mathrm{O}}^{2+}\right)$ value of $5.60 \mathrm{eV}$ at the oxygen-rich limit. In the previous GGA study, $\Delta E_{f}^{\mathrm{CBM}}\left(V_{\mathrm{O}}^{2+}\right)$ at the oxygen-rich limit is $8.46 \mathrm{eV}$ after the band-gap correction. With the PBEGGA, the band gap was underestimated by $\Delta E_{\mathrm{g}}=1.83 \mathrm{eV}$, and $\Delta E_{f}^{\mathrm{CBM}}\left(V_{\mathrm{O}}^{2+}\right)$ was raised by $3.66 \mathrm{eV}$ via the band-gap correction based on the rigid upward shift of the CBM. ${ }^{8}$ If the correction is not applied to the PBE-GGA and HSE06 results, $\Delta E_{f}^{\mathrm{CBM}}\left(V_{\mathrm{O}}^{2+}\right)$ are different by $\sim 0.2 \mathrm{eV}$. This points out that the CBM-shift-only scheme in conjunction with the GGA or LDA, showing severe band-gap underestimation, significantly overestimates defect formation energies relative to the HSE06 values. This can be partly attributed to the different absolute
VBM position obtained by respective functionals. Similar findings have been recently reported on other oxides such as $\mathrm{ZnO}$ (Refs. 16, 17, and 48), $\mathrm{TiO}_{2}$ (Ref. 18), and $\mathrm{In}_{2} \mathrm{O}_{3}{ }^{19}$

\section{SUMMARY}

The HSE06 hybrid functional calculations have been performed to study $V_{\mathrm{O}}$ in two perovskite tantalates, $\mathrm{KTaO}_{3}$ and $\mathrm{NaTaO}_{3}$. Their bulk properties such as the lattice constant and band gap are well reproduced using the HSE06 hybrid functional, and the calculated values are much closer to experimental data than those obtained by the GGA. The formation energies and single-particle electronic structures of $V_{\mathrm{O}}$ in $\mathrm{KTaO}_{3}$ and $\mathrm{NaTaO}_{3}$ indicate that $V_{\mathrm{O}}$ prefers to have $D_{4 h}$ symmetric atomic configuration and is a double shallow donor, which can provide carrier electrons for the $n$-type conductivity in both systems. Two other configurations, $V_{\mathrm{O}}-\mathrm{I}$ and $V_{\mathrm{O}}$-II, are also found through the symmetry breaking of atomic configurations near $V_{\mathrm{O}}$. These configurations possess the same point-group symmetry of $C_{4 v}$ but have very different single-particle electronic structures from each other. $V_{\mathrm{O}}$-II shows electron-localized states in the band gap, while $V_{\mathrm{O}^{-}}$ does not. However, these asymmetric configurations are less stable than the $D_{4 h}$ configuration, and thereby are unlikely to form in $\mathrm{KTaO}_{3}$ and $\mathrm{NaTaO}_{3}$ under thermal equilibrium. This explains no observations of $V_{\mathrm{O}}^{+}$in the EPR measurements and indicates that the high ionization energies assigned to $V_{\mathrm{O}}$ in electric conductivity measurements may not be responsible for $V_{\mathrm{O}}$ but for other defects, complexes, or impurities.

\section{ACKNOWLEDGMENTS}

This work was supported by Grants-in-Aid for Scientific Research (A), Young Scientists (B), Scientific Research on Priority Areas (Grant No. 474), and a Global COE Program from the Ministry of Education, Culture, Sports, Science, and Technology of Japan.

\footnotetext{
*choitheory@gmail.com

†oba@cms.mtl.kyoto-u.ac.jp

${ }^{1}$ K. Rabe, Ch. H. Ahn, and J.-M. Triscone (editors) Physics of Ferroelectrics: A Modern Perspective, Topics in Applied Physics (Springer, Berlin, 2007), Vol. 105.

${ }^{2}$ R. G. Geyer, B. Riddle, J. Krupka, and L. A. Boatner, J. Appl. Phys. 97, 104111 (2005).

${ }^{3}$ A.-K. Axelsson, Y. Pan, M. Valant, and N. Alford, J. Am. Ceram. Soc. 92, 1773 (2009).

${ }^{4}$ A. Sakai, T. Kanno, S. Yotsuhashi, H. Adachi, and Y. Tokura, Jpn. J. Appl. Phys. 48, 097002 (2009).

${ }^{5}$ H. Usui, S. Shibata, and K. Kuroki, Phys. Rev. B 81, 205121 (2010).

${ }^{6}$ H. Kato and A. Kudo, J. Phys. Chem. B 105, 4285 (2001).

${ }^{7}$ A. Shigemi, T. Koyama, and T. Wada, Phys. Status Solidi C 3, 1610 (2006).

${ }^{8}$ M. Choi, F. Oba, and I. Tanaka, Phys. Rev. B 78, 014115 (2008).
}

${ }^{9}$ I. P. Raevski, S. M. Maksimov, A. V. Fisenko, S. A. Prosandeyev, I. A. Osipenko, and P. F. Tarasenko, J. Phys. Condens. Matter 10, 8015 (1998).

${ }^{10}$ K. A. Müller, W. Berlinger, and R. S. Rubins, Phys. Rev. 186, 361 (1969).

${ }^{11}$ V. V. Laguta, M. D. Glinchuk, I. P. Bykov, A. Cremona, P. Galinetto, E. Giulotto, L. Jastrabik, and J. Rosa, J. Appl. Phys. 93, 6056 (2003).

${ }^{12}$ V. V. Laguta, M. I. Zaritskii, M. D. Glinchuk, I. P. Bykov, J. Rosa, and L. Jastrabík, Phys. Rev. B 58, 156 (1998).

${ }^{13}$ J. Heyd, G. E. Scuseria, and M. Ernzerhof, J. Chem. Phys. 118, 8207 (2003).

${ }^{14}$ J. Heyd, G. E. Scuseria, and M. Ernzerhof, J. Chem. Phys. 124, 219906 (2006)

${ }^{15}$ A. V. Krukau, O. A. Vydrov, A. F. Izmaylov, and G. E. Scuseria, J. Chem. Phys. 125, 224106 (2006).

${ }^{16}$ F. Oba, A. Togo, I. Tanaka, J. Paier, and G. Kresse, Phys. Rev. B 77, 245202 (2008) 
${ }^{17}$ J. L. Lyons, A. Janotti, and C. G. Van de Walle, Phys. Rev. B 80, 205113 (2009).

${ }^{18}$ A. Janotti, J. B. Varley, P. Rinke, N. Umezawa, G. Kresse, and C. G. Van de Walle, Phys. Rev. B 81, 085212 (2010).

${ }^{19}$ P. Ágoston, K. Albe, R. M. Nieminen, and M. J. Puska, Phys. Rev. Lett. 103, 245501 (2009).

${ }^{20}$ H.-P. Komsa and A. Pasquarello, Appl. Phys. Lett. 97, 191901 (2010).

${ }^{21}$ P. E. Blöchl, Phys. Rev. B 50, 17953 (1994).

${ }^{22}$ G. Kresse and J. Hafner, Phys. Rev. B 48, 13115 (1993).

${ }^{23}$ G. Kresse and J. Furthmüller, Phys. Rev. B 54, 11169 (1996).

${ }^{24}$ G. Kresse and D. Joubert, Phys. Rev. B 59, 1758 (1999).

${ }^{25}$ J. P. Perdew, K. Burke, and M. Ernzerhof, Phys. Rev. Lett. 77, 3865 (1996).

${ }^{26}$ F. Oba, M. Choi, A. Togo, A. Seko, and I. Tanaka, J. Phys. Condens. Matter 22, 384211 (2010).

${ }^{27}$ C. G. V. de Walle and J. Neugebauer, J. Appl. Phys. 95, 3851 (2004).

${ }^{28}$ S. Lany and A. Zunger, Phys. Rev. B 78, 235104 (2008).

${ }^{29}$ J. Ihm, A. Zunger, and M. L. Cohen, J. Phys. C 12, 4409 (1979).

${ }^{30}$ M. Leslie and N. J. Gillan, J. Phys. C 18, 973 (1985).

${ }^{31}$ G. Makov and M. C. Payne, Phys. Rev. B 51, 4014 (1995).

${ }^{32}$ S.-H. Wei, Comput. Mater. Sci. 30, 337 (2004).

${ }^{33}$ C. W. M. Castleton and S. Mirbt, Phys. Rev. B 70, 195202 (2004).

${ }^{34}$ J. Shim, E.-K. Lee, Y. J. Lee, and R. M. Nieminen, Phys. Rev. B 71, 035206 (2005).

${ }^{35}$ C. W. M. Castleton, A. Höglund, and S. Mirbt, Phys. Rev. B 73, 035215 (2006).

${ }^{36}$ J. P. Buban, H. Iddir, and S. Öğüt, Phys. Rev. B 69, 180102 (2004).
${ }^{37}$ K. Momma and F. Izumi, J. Appl. Crystallogr. 41, 653 (2008).

${ }^{38}$ M. D. Glinchuk, V. V. Laguta, I. P. Bykov, J. Rosa, and L. Jastrabik, J. Phys. Condens. Matter 7, 2605 (1995).

${ }^{39}$ A. P. Pechenyi, M. D. Glinchuk, C. B. Azzoni, F. Scardina, and A. Paleari, Phys. Rev. B 51, 12165 (1995).

${ }^{40}$ P. Grenier, G. Bernier, S. Jandl, B. Salce, and L. A. Boatner, J. Phys. Condens. Matter 1, 2515 (1989).

${ }^{41}$ S. Jandl, M. Banville, P. Dufour, S. Coulombe, and L. A. Boatner, Phys. Rev. B 43, 7555 (1991).

${ }^{42}$ M. Exner, H. Donnerberg, C. R. A. Catlow, and O. F. Schirmer, Phys. Rev. B 52, 3930 (1995).

${ }^{43}$ Y. S. Kim, D. J. Kim, T. H. Kim, T. W. Noh, J. S. Choi, B. H. Park, and J.-G. Yoon, Appl. Phys. Lett. 91, 042908 (2007).

${ }^{44}$ H. W. Jang, A. Kumar, S. Denev, M. D. Biegalski, P. Maksymovych, C. W. Bark, C. T. Nelson, C. M. Folkman, S. H. Baek, N. Balke, C. M. Brooks, D. A. Tenne, D. G. Schlom, L. Q. Chen, X. Q. Pan, S. V. Kalinin, V. Gopalan, and C. B. Eom, Phys. Rev. Lett. 104, 197601 (2010).

${ }^{45}$ M. Choi, F. Oba, and I. Tanaka, Phys. Rev. Lett. 103, 185502 (2009).

${ }^{46}$ G. Geneste, J.-M. Kiat, H. Yokota, Y. Uesu, and F. Porcher, Phys. Rev. B 81, 144112 (2010).

${ }^{47}$ J. Wen, G. Xu, C. Stock, P. M. Gehring, Z. Zhong, L. A. Boatner, E. L. Venturini, and G. A. Samara, Phys. Rev. B 78, 144202 (2008).

${ }^{48}$ S. J. Clark, J. Robertson, S. Lany, and A. Zunger, Phys. Rev. B 81, 115311 (2010). 